\title{
Bone marrow morphological features and diagnostic value in paediatric disseminated tuberculosis in the setting of increased HIV prevalence
}

\author{
R Grewal, MB ChB, FCPath (Haem), MMed; E A Abayomi, MBBS (Lond), MRCP (UK), FCPath (SA), MPhil, FRCP (Edin) \\ Division of Haematology, Department of Pathology, Faculty of Health Sciences, Stellenbosch University, Western Cape, South Africa
}

Corresponding author: R Grewal (rgrewal@sun.ac.za)

Background. Disseminated tuberculosis (TB) is a life-threatening condition which is often a challenge to diagnose. When to use bone marrow biopsies to diagnose disseminated TB in paediatrics is always a dilemma, from both a clinical and laboratory perspective, as there are no clear guidelines. Our study primarily aims to evaluate the role of routine bone marrow biopsies, and to compare peripheral blood cultures to aspirate cultures in the diagnosis of disseminated TB, in a paediatric population at Tygerberg Hospital. In addition, we set out to assess the morphology of bone marrow biopsies in this study.

Methods. A prospective study, consisting of 35 paediatric patients, was conducted from October 2007 to November 2008 . Bone marrow aspirate and trephine biopsies were performed on all patients and examined. Granulomas with Ziehl-Neelsen (ZN) positivity were sought on the trephine biopsy for the presence of acid-fast bacilli (AFB).

Results. Of the 35 children in this study, 25 were eventually diagnosed with TB on the basis of a multitude of clinical and laboratory parameters. The remaining 10 had alternative diagnoses. Peripheral blood TB cultures were positive in less than $1 \%$. Bone marrow aspirate cultures were positive in less than 5\%. Bone marrow trephine biopsies showed granulomas with ZN positivity in $11 \%$ of the 35 patients. Conclusion. Our results, generally, agree with the current evidence. Bone marrow biopsies in children should be performed if there is a strong clinical suspicion of disseminated $\mathrm{TB}$, when no alternative non-invasive confirmatory test is available.

S Afr Med J 2013;103(5):326-329. DOI:10.7196/SAMJ.6384

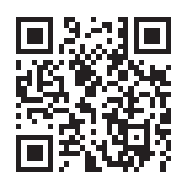

Tuberculosis (TB) is a major health problem worldwide, particularly in developing countries. One of the health targets set in the 2010 Millennium Development Goals is to halt and reverse the incidence of TB by 2015 ."

South Africa (SA) has one of the highest TB burdens in the world, ranked third after India and China. ${ }^{[2]}$ The spread of the TB epidemic is closely related to high HIV infection rates, particularly in subSaharan Africa, where $82 \%$ of TB-HIV co-infection is found. ${ }^{(3)}$

\section{Disseminated tuberculosis}

Disseminated TB is defined as TB that involves two or more noncontiguous sites. ${ }^{[t]}$ The clinical presentation may be chronic and nonspecific (with signs and symptoms such as fever, weight loss, anorexia and weakness) or acute (as fulminant disease). Clinical signs depend on organ involvement and, in order of frequency, include hepatomegaly, pulmonary findings, lymphadenopathy and splenomegaly. Children have a much higher risk of developing active TB, while disseminated TB is seen more frequently in children $<1$ year old. ${ }^{\text {[s] }}$

Disseminated TB requires accurate and early diagnosis, given that it is an increasingly significant cause of morbidity and mortality in developing countries, particularly in sub-Saharan Africa, where patients are likely to be co-infected with HIV. In areas where TB and HIV are endemic, TB septicaemia is among the leading causes of bloodstream infection. Moreover, TB is often neglected as a cause of death in cases of AIDS, ${ }^{[6]}$ where the mortality rate in children has been reported to be as high as $18-30 \%$.

The diagnosis of disseminated $\mathrm{TB}$ can be made on sputum or gastric washings through isolation of the Mycobacterium tuberculosis
$(M t b)$ bacilli. It may, however, be missed using these samples, and therefore more invasive investigations such as bronchoscopy, bone marrow biopsy and liver biopsy are required. The International Standards for TB Care recommend that, where the facilities and resources are available, appropriate specimens should be obtained for microscopy, culture and histopathological examinations from suspected sites of involvement in patients with presumed extrapulmonary/disseminated $\mathrm{TB}{ }^{|8|}$

Several factors obstruct diagnosis of disseminated TB in children. Obtaining sputum is difficult, leaving clinicians to resort to gastric aspirates. However, identification of acid-fast bacilli (AFB) on gastric aspirates is positive in less than $15 \%$ of samples, and only $30-40 \%$ are positive on culture.

The tuberculin skin test (TST) similarly has many limitations. While reactive in most children within $3-6$ weeks after initial infection, a positive reaction may be delayed for up to 3 months. False negative TSTs occur in up to $10 \%$ of children who are TB-culture positive, particularly in the context of severe TB illness, malnutrition, immunosuppression and the presence of other severe infections. Furthermore, asymptomatic infections caused by environmental non-tuberculous mycobacteria can give false positive results. ${ }^{[10]}$

Ker et al. ${ }^{[11]}$ conducted a prospective study in HIV-positive adult patients, comparing bone marrow studies with peripheral blood culture in the diagnosis of disseminated TB. They showed a higher diagnostic yield when bone marrow examination and peripheral blood culture were used in parallel. When disseminated TB is suspected in immune-competent adults, blood culture offers a safer, less invasive and cheaper investigation than bone marrow biopsy However, a positive blood culture is rare. ${ }^{[12]}$ Alternatively, a bone marrow aspirate can be cultured for AFB and other organisms. In 
addition, bone marrow trephine biopsy may show granulomas that may reveal AFB following Ziehl-Neelsen (ZN) staining. Positive bone marrow trephine results become available within $24-48$ hours. ${ }^{[13}$ However, in children, the paucibacillary nature of the disease makes detecting mycobacteria difficult.

To our knowledge, there has been only one prospective study comparing bone marrow investigation with peripheral blood culture in the diagnosis of disseminated TB in a paediatric cohort. ${ }^{[1 \mid}$ The study concluded that both modalities are useful in confirming disseminated $\mathrm{TB}$ in children.

\section{Methodology}

A prospective study was conducted from October 2007 to October 2008 at Tygerberg Hospital in Cape Town, in collaboration with the Division of Microbiology and Department of Paediatrics. Ethics approval was granted.

The study primarily aimed to evaluate the role of bone marrow biopsies in the diagnosis of disseminated TB in paediatric patients admitted to the hospital. Secondary aims were to compare peripheral blood cultures with aspirate cultures, identify AFB on trephine biopsies, compare the peripheral blood counts and morphological features of HIV-negative and -positive patients, and demonstrate the morphological findings of bone marrow biopsies in HIV-negative and -positive children.

Patients were recruited from Tygerberg Hospital and from outpatient clinics and referring hospitals, on the basis of being 13 years old or younger and having suspected disseminated TB. Consent was obtained from parents/legal guardians. Exclusion criteria included diagnosis of disseminated TB already confirmed by other means; lack of consent; severe illness and therefore inability to tolerate the bone marrow procedure; or showing signs of responding to TB treatment.

The Department of Paediatrics was responsible for taking histories and for physical examination, which included anthropometry and systemic examinations. The work-up of the patient encompassed Mantoux testing; chest X-ray; blood sampling for full blood counts, HIV test and blood culture; gastric washings and/or sputa for microscopy, culture and sensitivity and $\mathrm{ZN}$ stain; abdominal ultrasound; and cerebrospinal fluid analysis.

Peripheral blood and bone marrow aspirates and trephine biopsies were obtained under local anaesthetic and sedation. Samples were inoculated into BACTECMyco/F-Lytic bottles (BD, Franklin Lakes, NJ, USA) then subcultured into LöwensteinJensen solid media and incubated aerobically for 6 weeks. Cultures that were positive were confirmed as $M t b$ or other mycobacterial species by polymerase chain reaction (PCR). Slides of the peripheral blood and bone marrow aspirates were stained using Romanowsky and May-Grünwald Giemsa (MGG) stains respectively; the trephine biopsy was decalcified, fixed, embedded in wax, cut, trimmed and stained using haematoxylin and eosin (H\&E), MGG and reticulin stains. Each trephine biopsy specimen was examined to assess cellularity, haematopoietic maturation, and for presence of dysplastic changes, stromal changes, granulomas or any other pathology. ZN staining was performed on biopsies containing granulomas to identify AFB.

\section{Results}

A total of 45 patients with suspected disseminated TB were evaluated for the study. Ten were excluded, 8 because consent was not obtained and 2 following confirmation of disseminated TB by other means. Thus, 35 patients were enrolled. The diagnosis of TB was confirmed in 25 patients, 19 of whom had positive cultures (gastric washings/ sputum, blood or aspirate) and/or granulomas on histology (Table 1), while 6 patients were classified as having probable TB and treated as such, based on clinical suspicion and miliary pattern on chest X-ray, but in the absence of laboratory confirmation. Of the 25 patients with mycobacterial disease, 20 were diagnosed with disseminated TB while 5 had TB confined to a single site. Mtb was the causative agent in $84 \%(16 / 19)$ of confirmed cases.

Granulomas were identified in $11 \%(5 / 35)$ of all bone marrow trephine biopsies (Fig. 1), 4 of which showed ZN positivity, and in $20 \%$ (5/25) of HIV-positive patients who were subsequently confirmed to have TB. Of note, $81 \%(22 / 25)$ of patients diagnosed with TB had already started TB treatment at time of bone marrow biopsy. Meanwhile, $22 \%$ of HIV-positive patients showed granulomas on trephine biopsy compared with $10 \%$ in the HIV-negative population (Figs 2 and 3).

Peripheral blood cultures were positive in less than $1 \%(1 / 35)$ and bone marrow aspirate cultures were positive in less than 5\% (3/35) of the patients (Fig. 4). An HIV test was not performed in $6 \%(2 / 35)$ of patients, $60 \%(21 / 35)$ tested negative for HIV, and 34\% (12/35) were positive (Fig. 5).

Anaemia, thrombocytosis/thrombocytopenia were the main peripheral blood findings: anaemia in $26 \%(9 / 35)$, thrombocytosis in $17 \%(6 / 35)$ and thrombocytopenia in 17\% (6/35). In HIV-positive patients, peripheral blood findings were as follows: anaemia (25\%), pancytopenia (25\%), thrombocytopenia (25\%), bicytopenia (17\%), and leukopenia (8\%). In contrast, HIV-negative patients presented with anaemia (27\%), thrombocytosis (27\%), thrombocytopenia (27\%), bicytopenia (14\%) and pancytopenia (9\%). Of the HIVnegative patients, $9 \%$ had normal counts. No patients in the HIVpositive group showed normal counts or thrombocytosis (Table 2).

The peripheral blood morphology of our study group showed the following: $46 \%$ showed features of infection, $29 \%$ were normal and $14 \%$ showed dysplastic features.

Of the HIV-positive patients, $42 \%$ showed dysplasia; no dysplasia was noted in the peripheral blood smear of HIV-negative patients (Table 3).

The bone marrow biopsy morphological features were as follows: $51 \%$ were normal (18/35), $25 \%$ showed secondary myelodysplasia (9/35), 10\% showed mild dysplastic features (4/35), 3\% aplastic anaemia (1/35), and $3 \%$ showed eosinophilia (1/35). In $3 \%(1 / 35)$, the biopsy was suboptimal for interpretation, and in $3 \%(1 / 35)$ examination was not carried out. Of the patients with HIV, 64\% showed HIV-associated myelodysplasia; myelodysplastic features were not seen in any of the HIV-negative patients (Figs 2 and 3). No complications related to the bone marrow occurred. Bone marrow trephine biopsy results were communicated to the clinician within 48 hours of the procedure. TB was excluded confidently in 10 patients.

Table 1. Comparison of the diagnostic yield of the specimens collected for the diagnosis of TB

\begin{tabular}{lllll}
\hline Total patients $(N)$ & $\begin{array}{l}\text { Gastric washings } \\
\text { culture positive }(n)\end{array}$ & $\begin{array}{l}\text { Peripheral blood } \\
\text { culture positive }(n)\end{array}$ & $\begin{array}{l}\text { Aspirate culture } \\
\text { positive }(n)\end{array}$ & ZN-positive granulomas $(n)$ \\
\hline 35 & 17 & 1 & 3 & 4
\end{tabular}




\section{RESEARCH}

Table 2. Comparison of peripheral blood counts of HIVnegative and HIV-positive patients

\begin{tabular}{lll}
\hline & HIV-negative (\%) & HIV-positive (\%) \\
\hline Normal & 9 & 0 \\
Anaemia & 27 & 25 \\
Thrombocytopenia & 14 & 25 \\
Leucopenia & 0 & 8 \\
Bicytopenia & 14 & 17 \\
Pancytopenia & 9 & 25 \\
Thrombocytosis & 27 & 0
\end{tabular}

Table 3. Comparison of peripheral blood morphology features

\begin{tabular}{lll}
\hline & HIV-negative (\%) & HIV-positive (\%) \\
\hline Normal & 43 & 8 \\
Features of infection & 44 & 50 \\
Dysplastic features & 0 & 42 \\
Not done & 13 & 0
\end{tabular}

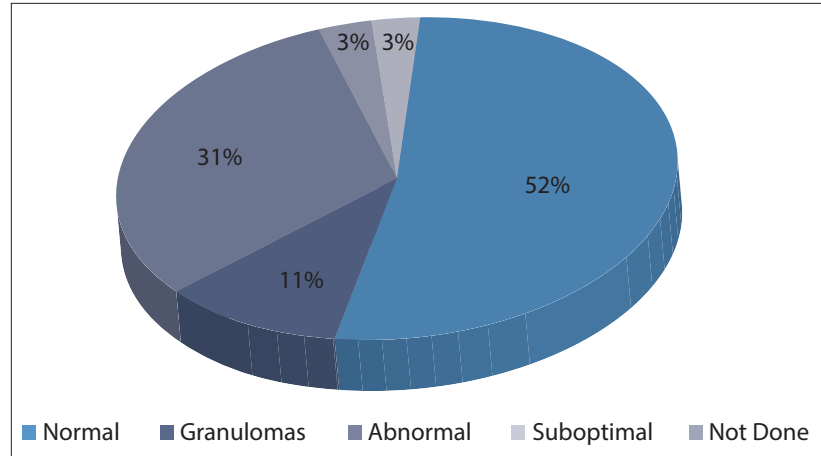

Fig. 1. Granulomas on trephine biopsies: $11 \%$ of the patients showed granulomas on the trephine biopsy.

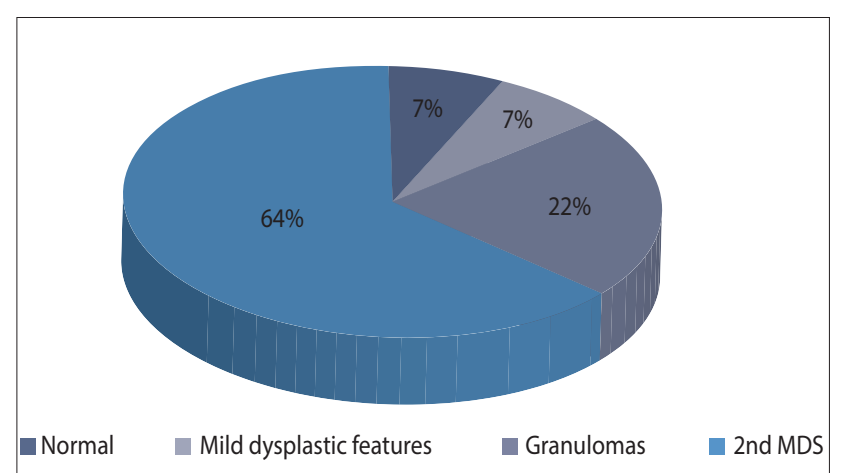

Fig. 2. Bone marrow morphology findings of HIV-positive patients: $64 \%$ of patients with HIV showed HIV-related myelodyplastic changes (2nd MDS).

\section{Discussion}

Because disseminated TB is fatal if not diagnosed early, strong clinical suspicion of the condition, confirmation of the diagnosis and urgent institution of treatment are necessary. As the diagnosis of disseminated $\mathrm{TB}$ is often missed on sputum, more invasive investigations are required. ${ }^{[]}$Unfortunately, our study sample was too small to show any statistical significance. Disseminated TB was diagnosed on the basis

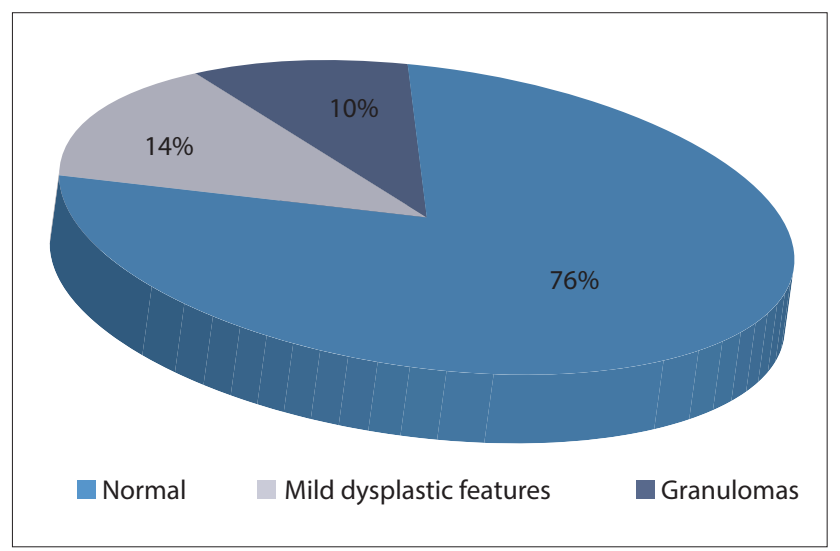

Fig. 3. Bone marrow morphology findings in HIV-negative patients: $76 \%$ of HIV-negative patients had normal marrows.

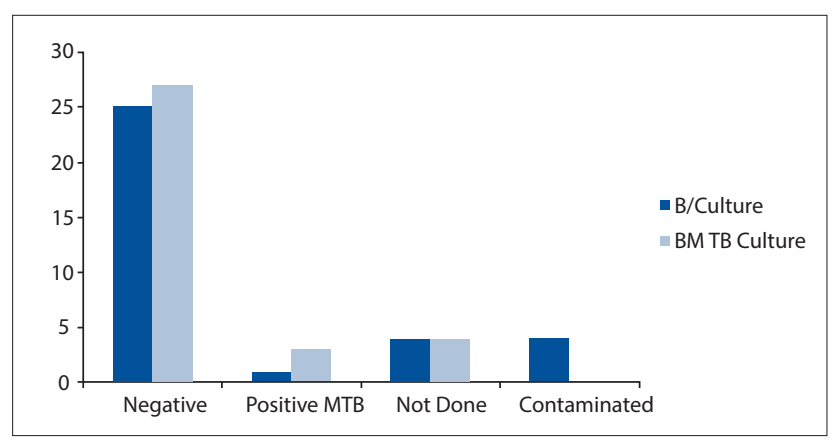

Fig. 4. Comparison of peripheral blood cultures (B/culture) and bone marrow aspirate cultures (BM TB culture): most samples were negative for $T B$.

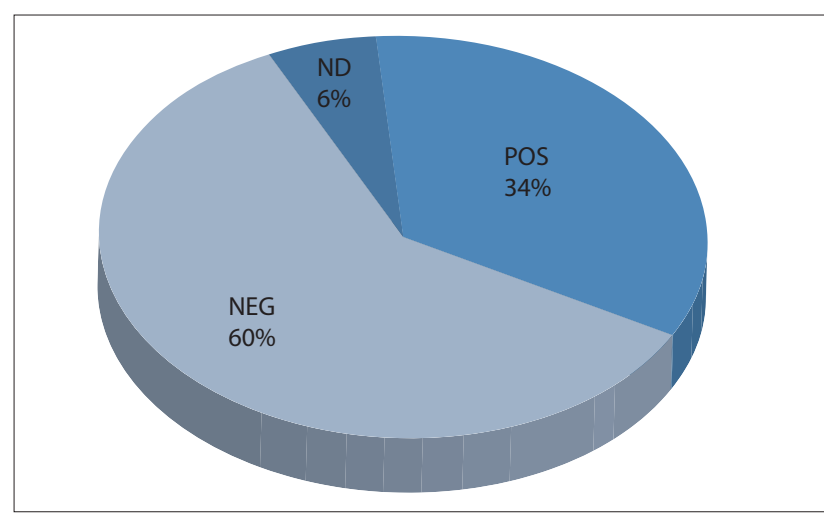

Fig. 5. HIV status of patient cohort: 34\% (12/35) of patients were HIV-positive, $60 \%$ (21/35) were HIV-negative and 6\% (2/35) were not tested for HIV.

of the presence of granulomas in $11 \%$ of patients undergoing trephine biopsy. The yield was even greater, at $20 \%$, in those patients who were HIV-positive and in whom TB was finally confirmed.

The main disadvantage of our study was that most patients had already started TB treatment when bone marrow biopsy was performed. According to Rose et al., respiratory specimens sent for culture before treatment is initiated provide the best mycobacterial culture yield at $49 \%$; the diagnostic yield of the trephine biopsy is much lower in comparison. ${ }^{\mid \text {|s| }}$ We therefore speculate that the low rate of detection of AFB on the trephine biopsy might have been influenced by our patients starting treatment before referral for a bone marrow biopsy, rather than risk delay in commencing treatment for disseminated TB. Rose et al. noted also that the 4- to 6-week 
waiting period for positive $M t b$ culture results was too prolonged to influence TB management. ${ }^{15 \mid}$ In contrast, in our study, results of the bone marrow trephine biopsy were available within 48 hours and therefore treatment with anti-TB drugs could be implemented immediately in 4 patients.

David et al. assessed the role of peripheral blood cultures in the diagnosis of adult TB, and confirmed TB in 19\% (15/80) of their patients. ${ }^{\left[{ }^{161}\right.}$ In our study, in comparison, peripheral blood and bone marrow aspirate cultures showed an extremely low $M t b$ yield. The lack of standardisation for collecting these samples, the variety of clinical personnel involved in sample collection and the paucibacillary nature of the disease in children are all underlying explanations for this very low $M t b$ yield. Evidence suggests that bone marrow aspirate cultures are more sensitive than peripheral blood cultures for the diagnosis of disseminated TB. ${ }^{(1.1)}$ We also demonstrated that complete evaluation of bone marrow aspirate and trephine biopsies provides a higher diagnostic yield.

As further support for performing a bone marrow biopsy, we were able to provide an explanation for the cytopenias in our HIV-positive patients. HIV-related myelodysplasia is a well-known phenomenon and is known to contribute towards cytopenias. ${ }^{[1]}$ The majority of our HIV-positive patients were diagnosed with an HIV-related secondary myelodysplastic syndrome, while none of our HIV-negative patients showed such features. In addition, our HIV-positive patients showed a higher percentage of granulomas than those who were HIV-negative.

Cucin et al. compared the roles of liver biopsy and bone marrow biopsy in the diagnosis of disseminated TB, and concluded that bone marrow biopsy was less hazardous. ${ }^{[1 \mid}$ We can recommend bone marrow trephine and biopsy as a safe procedure since no complications occurred in our patients.

\section{Conclusion}

In conclusion, a low threshold for undertaking bone marrow aspirate and trephine is recommended to confirm the diagnosis in patients in whom HIV positivity, cytopenias, organomegaly and fever arouse suspicion of disseminated TB.

Acknowledgements. We wish to thank our patients and their parents/ guardians, Dr Riezah Abrahams from the Tygerberg Hospital Department of Microbiology; Dr Penelope Rose from the Tygerberg Hospital Department of Paediatrics; doctors who referred patients to the study and the doctors who assisted with analysing samples from bone marrow biopsies.

\section{References}

1. World Health Organization. The Global Plan to Stop TB, 2011-2015. Geneva: World Health Organization, 2010. http://www.stoptb/org/global/plan

2. World Health Organization. WHO 2010 report: Global TB control. Geneva: World Health Organization, 2010. http://www.who.int/tb/publications/global report/2010

World Health Organization. WHO (2011) TB/HIV facts 2011 (Fact sheet) Geneva: World Health Organisation, 2011. http://www/whqlibdoc.who.int/publications/2011

Miyoshi I, Daibata M, Kuroda N, Taguchi H, Enzan H. Miliary tuberculosis not affecting the lungs but complicated by acute respiratory distress syndrome. Intern Med 2005;44(6):622-624.

5. Eamranond P, Jaramillo E. Tuberculosis in children: reassessing the need for improved diagnosis in global control strategies. Int J Tuberc Lung Dis 2001;5(7):594-603.

6. Crump JA, Reller LB. Two decades of disseminated tuberculosis at a university medical center: the expanding role of mycobacterial blood culture. Clin Infect Dis 2003;37(8):1037-1043. [http://dx.doi. org/10.1086/378273

Maartens G, Willcox PA, Benatar SR. Miliary tuberculosis: rapid diagnosis, hematologic d outcome in 109 treated adults. Am J Med 1990;89(3):291-296.

. Hopewell PC, Pai M, Maher D, Uplekar M, Raviglione MC. International standards for tuberculosis care. Lancet Infect Dis 2006;6(11):710-725. [http://dx.doi.org/10.1016/S1473-3099(06)70628-4]

Cruz AT, Starke JR. Clinical manifestations of tuberculosis in children. Paediatr Respir Rev 2007;8(2):107-117. [http://dx.doi.org/10.1016/j.prrv.2007.04.008]

10. Shingadia D, Novelli V. Diagnosis and treatment of tuberculosis in children. Lancet Infect Dis 2003;3(10):624-632

11. Cheng-Chin Ker, Chien-Ching Hung, Shang-Yee Huang, et al. Comparison of bone marrow studies with blood culture for etiological diagnosisof disseminated mycobacterial and fungal infection in patients with acquired immunodeficiency syndrome. J Microbiol Immunol Infect 2002;35(2):89-93 12. Kramer F, Modilevsky T, Waliany AR, Leedom JM, Barnes PF. Delayed diagnosis of tuberculosis in patients with human immunodeficiency virus infection. Am J Med 1990;89(4):451-456.

13. Bain B, Clark DM, Lampert IA, Wilkins BS. Bone Marrow Pathology, 3rd ed. Hoboken: Blackwell Sciences, 2005

14. Kimsey LS. The bacteriological diagnosis of childhood tuberculosis using bone marrow and peripheral blood aspirates. J Pediatr 1958;53:413-431

15. Rose PC, Schaaf HS, Marais BJ, Gie RP, Stefan DC. Value of bone marrow biopsy in children with suspected disseminated mycobacterial disease. Int J Tuberc Lung Dis 2011;1592:200-204

16. David ST, Mukundan U, Brahmadathan KN, John TJ. Detecting mycobacteraemia for diagnosing tuberculosis. Indian J Med Res 2004;119(6):259-266.

17. Tripathi AK, Kalra P, Misra R, Kumar A, Gupta N. Study of bone marrow abnormalities in patients with HIV disease. J Assoc Physicians India 2005;53:105-110.

18. Cucin RI, Coleman M, Eckardt JJ, Silver RT. The diagnosis of military tuberculosis: utility of peripheral blood abnormalities, bone marrow and liver needle biopsy. J Chronic Dis 1973, 26(6):355-361

Accepted 24 October 2012 